TITLE:

\title{
Effects of the guard electrode on the photoelectron distribution around an electric field sensor
}

$\operatorname{AUTHOR}(\mathrm{S})$ :

Miyake, Y.; Usui, H.; Kojima, H.

\section{CITATION:}

Miyake, Y....[et al]. Effects of the guard electrode on the photoelectron distribution around an electric field sensor. JOURNAL OF GEOPHYSICAL RESEARCH-SPACE PHYSICS 2011 , 116(A5): A05211.

\section{ISSUE DATE:}

2011-05

URL:

http://hdl.handle.net/2433/163425

RIGHT:

(C)2011. American Geophysical Union. 


\title{
Effects of the guard electrode on the photoelectron distribution around an electric field sensor
}

\author{
Y. Miyake, ${ }^{1}$ H. Usui, ${ }^{2}$ and H. Kojima ${ }^{3}$ \\ Received 30 April 2010; revised 14 February 2011; accepted 18 February 2011; published 18 May 2011.
}

[1] We have developed a numerical model of a double-probe electric field sensor equipped with a photoelectron guard electrode for the particle-in-cell simulation. The model includes typical elements of modern double-probe sensors on, e.g., BepiColombo/ MMO, Cluster, and THEMIS spacecraft, such as a conducting boom and a preamplifier housing called a puck. The puck is also used for the guard electrode, and its potential is negatively biased by reference to the floating spacecraft potential. We apply the proposed model to an analysis of an equilibrium plasma environment around the sensor by assuming that the sun illuminates the spacecraft from the direction perpendicular to the sensor deployment axis. As a simulation result, it is confirmed that a substantial number of spacecraft-originating photoelectrons are once emitted sunward and then fall onto the puck and sensing element positions. In order to effectively repel such photoelectrons coming from the sun direction, a potential hump for electrons, i.e., a negative potential region, should be created in a plasma region around the sunlit side of the guard electrode surface. The simulation results reveal the significance of the guard electrode potential being not only lower than the spacecraft body but also lower than the background plasma potential of the region surrounding the puck and the sensing element. One solution for realizing such an operational condition is to bias the guard potential negatively by reference to the sensor potential because the sensor is usually operated nearly at the background plasma potential.

Citation: Miyake, Y., H. Usui, and H. Kojima (2011), Effects of the guard electrode on the photoelectron distribution around an electric field sensor, J. Geophys. Res., 116, A05211, doi:10.1029/2010JA015600.

\section{Introduction}

[2] In scientific spacecraft missions, the double-probe technique is commonly used for electric field measurements covering a wide frequency range, from DC to above the local electron plasma and cyclotron frequencies [Fahleson, 1967; Pedersen et al., 1984; Tsuruda et al., 1994; Pedersen et al., 1998; Gurnett, 1998]. Although the fundamental principle of the measurement technique is relatively simple, careful attention should be paid for the sensor coupling with a surrounding plasma environment affected by photoelectron emission, which occasionally influences the sensor performance.

[3] A great deal of research has been conducted over the past few decades with the goal of improving the doubleprobe technique in order to further enhance the reliability and accuracy of electric field measurements. One improved

\footnotetext{
${ }^{1}$ Academic Center for Computing and Media Studies, Kyoto University, Kyoto, Japan.

${ }^{2}$ Graduate School of System Informatics, Kobe University, Kobe, Japan.

${ }^{3}$ Research Institute for Sustainable Humanosphere, Kyoto University, Kyoto, Japan.

Copyright 2011 by the American Geophysical Union. 0148-0227/11/2010JA015600
}

technique involves the application of the "hockey puck" principle, where the "hockey puck" (or shortly "puck") indicates a preamplifier housing separated from both a spacecraft body and a spherical probe. The principle is used practically in the Cluster Electric Fields and Waves (EFW) instrument [Gustafsson et al., 1997, 2001]. According to the Cluster Active Archive (available at http://caa.estec.esa.int/ documents/UG/CAA_EST_UG_EFW_v30.pdf), Cluster EFW has a typical accuracy of $\sim 0.5 \mathrm{mV} / \overline{\mathrm{m}}$ in static electric field measurements. Then, the continuous improvements of the hockey puck principle have been made through its use for the THEMIS Electric Field Instrument (EFI) [Bonnell et al., 2008]. At present, there are plans to apply the principle to the Mercury Electric Field In-Situ Tool (MEFISTO) for the BepiColombo/MMO spacecraft to Mercury [Blomberg et al., 2006].

[4] The puck is typically extended from the spacecraft by means of a conducting boom, and a sensing element composed of a spherical probe with a thin wire is deployed outward from the puck. The primary purpose of the puck is to locate preamplifiers close to the sensing elements for optimum DC electric field measurement. In case of MEFISTO, the puck is also used as a guard against photoelectrons coming from the spacecraft body. For this purpose, the puck surface potential is operationally controlled so as to have 
exact values with respect to the spacecraft body or a sensing element of the instrument.

[5] Since spacecraft-originating photoelectrons are recognized as interfering with electric field measurements [Pedersen et al., 1998], the photoelectron guard implementation has been an important technique on recent magnetospheric missions. However, because of the voltage applied to the photoelectron guard electrode and the peculiar geometry of the whole instrument, the photoelectron and potential distributions around the sensor are so complicated. In order to further improve the sensor performance for BepiColombo/MMO and other future magnetospheric missions, it is necessary to better understand the plasma environment surrounding the sensor depending on operating conditions of the guard electrode.

[6] Several numerical investigations have been performed to examine the characteristics of the hockey puck instrument and its surrounding environments. Béghin et al. [2005] developed a numerical model of Cluster EFW based on the Surface Charge Distribution (SCD) method and presented its electric properties in a high-frequency range, excluding the effect of a photoelectron cloud. Cully et al. [2007] reported the electrostatic potential structure around Cluster EFW considering the kinetics of photoelectrons. Their approach puts emphasis on the modeling of realistic sensor dimensions by limiting their analysis target to steady state solutions. Consequently, although the numerical tool includes some modeling and assumptions for, e.g., a treatment of the floating potentials of the spacecraft and instrument, their approach has shown its practical value in producing quantitative knowledge about an equilibrium photoelectron distribution around the sensor.

[7] On the other hand, we consider another approach to understand the behavior of the instrument, i.e., to stress the self-consistency of a system in order to enable the method to be used to analyze a wide range of possible situations such as including transient variations in sensor potentials. For example, a sensor receiving AC electric fields corresponds to such a situation. There is an urgent need to develop a numerical tool based on the latter approach for comprehensive understanding of the sensor behavior from DC to high-frequency ranges.

[8] To meet with this requirement, we applied particle-incell (PIC) simulations to the analysis of plasma processes emerging around the electric field sensor. The Electromagnetic Spacecraft Environment Simulator (EMSES) proposed in a previous paper is based on the electromagnetic PIC method combined with a numerical treatment of internal boundaries describing the conducting spacecraft and sensor bodies [Miyake and Usui, 2009]. In principle, the use of EMSES can provide self-consistent solutions of plasma and photoelectron behaviors as well as the transient variation of spacecraft and sensor potentials. Meanwhile, we should recognize the difficulty of including realistic sensor dimensions and plasma parameters resulting from the high computational cost of EMSES.

[9] Considering both the above advantages and limitations, we herein deal with (1) the numerical modeling of the double-probe electric field sensor equipped with a photoelectron guard electrode and (2) the behavior of the photoelectron guard electrode and its influence on the equilibrium photoelectron distribution around the sensor. We especially focus on the distribution of spacecraft-originating photoelectrons. Although the spacecraft-originating photoelectrons arriving at the sensing element position are only a very small fraction of the total photoelectron outflow, if we consider a high-density ratio of the photoelectrons compared to tenuous magnetospheric plasmas, there is a significant possibility of influencing the current balance formed at the sensing element of the instrument. Hence, the dependence of the spacecraft-originating photoelectron distribution on operating conditions of the guard electrode deserves systematic investigations using the PIC approach.

[10] In section 2, we describe a numerical model of the sensor, specifically models of the puck surface potential control to realize the guard electrode operation and the current biasing. Section 3 presents simulation results for the equilibrium plasma environment around the sensor. We first present an overall picture of the photoelectron distribution around the sensor and then clarify how the guard electrode prevents spacecraft-originating photoelectrons from approaching a sensing element location. Section 4 presents discussions about the achievements and limitations of this work. Finally we conclude the present study in section 5 .

\section{Numerical Model}

\subsection{Simulation Code}

[11] We use the simulation code called EMSES, which was originally developed for the self-consistent analysis of spacecraft-plasma interactions on the full PIC basis. As with the conventional particle code, EMSES has a grid system, on which any field components are defined, and a large number of macroparticles, which represent plasma and can take arbitrary positions [Birdsall and Langdon, 1985]. We solve Maxwell's equations governing the electromagnetic field evolution and Newton's equations of motion for each particle in a simultaneous manner.

[12] Solid spacecraft and sensor bodies are represented as boundaries of conducting surfaces located inside a computational domain. Both longitudinal and transverse electric fields on the inner boundaries should satisfy conducting surface conditions correctly. For the longitudinal component, we handle the charge accumulation due to plasma impinging into and emission from the conducting surfaces by adopting a special boundary treatment for current density [Miyake and Usui, 2009]. We then redistribute the surface charge based on the capacity matrix method to realize an equipotential solution on the boundaries [Hockney and Eastwood, 1981]. After these treatments, only a transverse component remains on the boundaries. We can simply eliminate the remained transverse electric field, which completes the conducting boundary treatment.

[13] The particle emission, e.g., photoemission, from the surfaces is modeled using a conventional particle-loading scheme [Cartwright et al., 2000]. Based on the number of emitted particles per one time step and their energy given as input parameters, the subroutine supervising particles computes the initial positions and velocities of the emitted particles at every time step, and then begins to solve their motion. The particles are emitted in such a manner that charges of the same quantity and opposite sign of the par- 

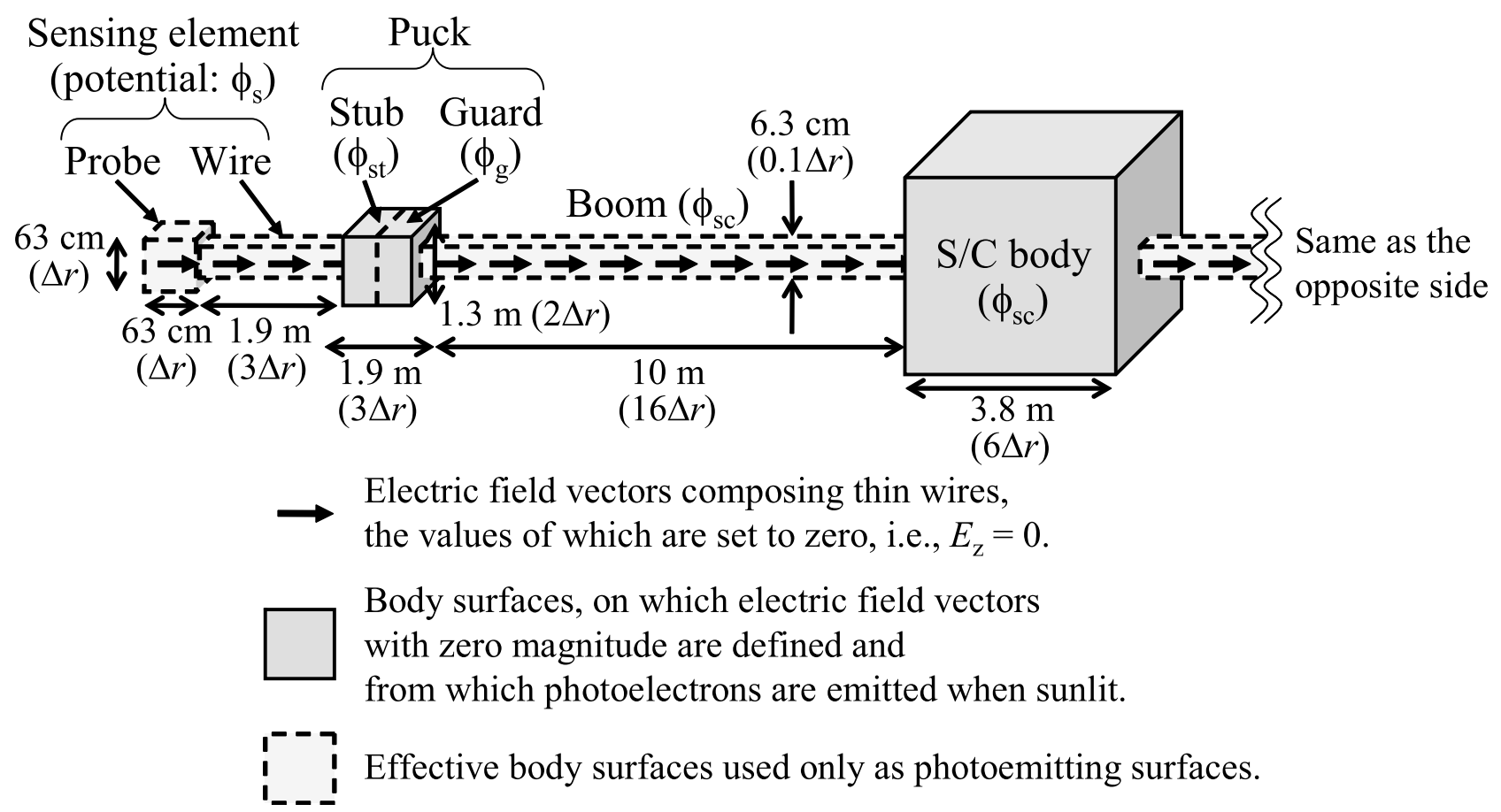

Figure 1. Configuration and dimensions of the numerical model of the electric field sensor used in the present study. Parenthetical symbols $\left(\right.$ e.g., $\phi_{\mathrm{s}}$ ) displayed below the conducting element names are used for their potentials in the present paper. We define the stub and guard voltages as $V_{\mathrm{st}-\mathrm{s}}=\phi_{\mathrm{st}}-\phi_{\mathrm{s}}$ and $V_{\mathrm{g} \text {-sc }}=$ $\phi_{\mathrm{g}}-\phi_{\mathrm{sc}}$, respectively. The tip-to-tip length of the sensor corresponds to $52 \Delta r$.

ticles correctly remain at the spacecraft surfaces. The current version of the simulation code supports a single Maxwellian for the velocity distribution of the emitted particles.

[14] The validity of EMSES were discussed in detail in a previous paper [Miyake and Usui, 2009]. Another important consideration is that the present code supports only uniform, Cartesian coordinate grid systems. This constraint introduces limitations in modeling the sensor configuration addressed in section 2.2 .

\subsection{Modeling of Sensor Structure}

[15] Figure 1 illustrates the sensor model used in the present simulation. Its overall structure is determined based on the latest design of the double-probe electric field sensor, i.e., MEFISTO for the BepiColombo/MMO spacecraft. MEFISTO can be roughly divided into four components: a spherical probe, a thin wire, a cylindrical boom, and a preamplifier housing referred to as a puck [Blomberg et al., 2006]. One sensing element is composed of the spherical probe and thin wire, the surfaces of which have the same electric potential. The boom surface is coated by a conducting material and is connected electrically to the spacecraft ground. The puck is then situated between the sensing element and the boom.

[16] The puck also functions as a photoelectron guard electrode. Its external surface consists of two parts that are electrically insulated from each other. The inner (boomside) and outer (sensorside) surfaces of the puck are referred to as a guard and a stub, respectively. Then, the electrical potentials of the guard and stub are controlled independently. The guard is installed for the purpose of repelling photoelectrons emitted from the spacecraft body and pre- venting them from approaching the sensing element, whereas the stub is installed for the purpose of attracting photoelectrons emitted from the sensing element. The operational potential control of the guard and stub will be described in detail later herein.

[17] Based on the above design, we constructed the model shown in Figure 1. The model includes the spacecraft body and a pair of electric field sensors. Since we must represent the geometry using a limited number of rectangular grid elements, certain components must be deformed considerably from the actual MEFISTO design.

[18] Our baseline assumption is that the spacecraft body and the pucks are rectangular. The sensor wires and the boom cylinders are represented electrically by a single column of computational grid points, as used by Miyake et al. [2008]. More concretely, we impose an equipotential condition over the grid points on the single column, and also ensure that electric field vectors defined between the grid points have zero magnitudes. One problem with this wire modeling manner is that the thickness of the wire becomes unclear. According to the literature of the contour path FDTD modeling [Taflove, 1995], a wire modeled in this manner is interpreted to have an equivalent radius of $\exp (-2) \Delta r \sim 0.14 \Delta r$, where $\Delta r$ represents the grid spacing.

[19] According to this rule, in order to model the practical radius of the sensor wire, e.g., $\sim 0.1 \mathrm{~mm}$ for MEFISTO, we must set $\Delta r \sim 1 \mathrm{~mm}$. However, if we set $\Delta r \sim 1 \mathrm{~mm}$ in a uniform grid system, a simulation containing the entire spacecraft and its surrounding plasmas is impractical in terms of computational cost. Therefore, we instead set $\Delta r$ to be comparable to the shielding length by photoelectrons (typically $10-10^{2} \mathrm{~mm}$ ), which results in much thicker wire 
elements as compared to the MEFISTO configuration. Also, the radius of a spherical probe, $40 \mathrm{~mm}$ for MEFISTO, is comparable to or even smaller than $\Delta r$, which makes it difficult to include the spherical structure of the probe.

[20] For the simulation of photoelectron emission and particle absorption at the bodies, we also need to define solid surfaces that are sensed by macroparticles. For the spacecraft and puck bodies, the solid surfaces accord with mesh surfaces on which perfect conducting conditions for electric field are imposed. However, for the sensor and boom wires, we cannot represent the solid surfaces by the mesh. This is because the wires are electrically defined by only a single column of grid points as described above, which implies that their cross sections are much smaller than $\Delta r^{2}$. Although such wires appear to have infinitesimal radius and surface area, it would be more appropriate to assume that the wires have a finite effective radius, because charges defined on the grid points should be interpreted to have some broadening around the grid points. Thus, we define effective solid surfaces for the sensor and boom wires, as illustrated in Figure 1. We use $0.1 \Delta r$ as the wire effective radius, regulated by the delimitation of the particle push resolution as determined by the time step width $\Delta t$. Meanwhile, we assume the effective thickness of $\Delta r$ for the end of the sensor wire corresponding to the spherical probe.

\subsection{Potential Control of Sensor Surfaces}

[21] According to the baseline specifications, MEFISTO is supposed to be used under the following conditions: (1) the two opposite sensing elements have about the same voltage between their surface and local plasma potentials, (2) the spacecraft body is in a floating potential condition, (3) the stub surface is a few V positive with respect to the sensing element, and (4) the guard surface is $\sim 10 \mathrm{~V}$ negative with respect to the spacecraft body [Blomberg et al., 2006]. The proposed numerical model is also based on the same philosophy.

[22] In principle, the magnitude of the guard and stub potentials should be large enough to change photoelectron orbits. Considering the typical energy of the photoelectrons, i.e., a few $\mathrm{eV}$, we determine the guard and stub potentials to be $-8 \phi_{\mathrm{ph}}$ and $+2 \phi_{\mathrm{ph}}$ with respect to the spacecraft and sensing element, respectively. Here, the potential $\phi_{\mathrm{ph}}$ multiplied by the electric charge unit $e$ corresponds to the most probable photoelectron energy.

[23] In addition, a certain magnitude of current is provided as a bias current from the spacecraft body to the sensing element. The current value is given as an input parameter $I_{\text {bias. }}$. The purpose of the current biasing is to maintain the sensor potential close to the background space potential. This condition produces the low-contact resistance of the probes with respect to the local plasmas, which is ideal for electric field measurements based on a voltmeter principle [Tsuruda et al., 1994]. The problem is that the optimum $I_{\text {bias }}$ value necessary to accomplish this purpose is unknown. In the present study, since we can measure the potential difference between the sensing element and the background space directly from simulation outputs, we choose an appropriate magnitude after examining several simulations for various values of $I_{\text {bias. }}$. Meanwhile, from a practical standpoint, the $I_{\text {bias }}$ magnitude is roughly determined by analytical estimation using typical or experimentally obtained plasma parameters [Tsuruda et al., 1994], so that the sensor potential may not have a negative value. More technically, the optimum $I_{\text {bias }}$ value can also be estimated from a current-voltage characteristic of the sensor using the inflection point method as an extension of the Langmuir probe theory [Hershkowitz, 1989; Smith et al., 1979].

[24] We implement the above functions as an extension of the capacity matrix method [Hockney and Eastwood, 1981; Miyake and Usui, 2009]. The capacity matrix provides the quantitative relation between the electric potentials and charges on the conducting surfaces. The general purpose of the capacity matrix method is to compute the longitudinal electric fields so as to satisfy the equipotential condition for each body. In the current model, we additionally introduce special constraint conditions for the charges and potentials in order to properly simulate the puck potential control and current biasing. The use of the capacity matrix method can consistently ensure the conservation of the total charge of the entire instrument and spacecraft bodies.

[25] We first summarize the known parameters and the constraint conditions used in the capacity matrix processing. As input parameters, we have information concerning $I_{\text {bias }}$, $V_{\mathrm{st}-\mathrm{s}}=\phi_{\mathrm{st}}-\phi_{\mathrm{s}}$, and $V_{\mathrm{g}-\mathrm{sc}}=\phi_{\mathrm{g}}-\phi_{\mathrm{sc}}$, where $\phi$ represents the conducting body potential, and the subscripts "st", "s", "g", and "sc" correspond to the stub, the sensing element, the guard, and the spacecraft body (including the boom), respectively. $V_{\text {st-s }}$ and $V_{\mathrm{g}-\mathrm{sc}}$ represent the stub and guard voltages, i.e., the stub-to-sensor and guard-to-spacecraft potentials, respectively. In addition to the parameters, the following charge conservation conditions should be satisfied at all times and should be used in the capacity matrix processing:

$$
\begin{gathered}
\Delta Q_{\mathrm{s} 1}=I_{\text {bias }} \Delta t, \\
\Delta Q_{\mathrm{s} 2}=I_{\text {bias }} \Delta t, \\
\Delta Q_{\mathrm{g} 1}+\Delta Q_{\mathrm{g} 2}+\Delta Q_{\mathrm{st} 1}+\Delta Q_{\mathrm{s} 2}+\Delta Q_{\mathrm{sc}}=-2 I_{\text {bias }} \Delta t,
\end{gathered}
$$

where $\Delta Q$ represents the charge variation during $\Delta t$ on one conducting element. Since elements other than the spacecraft body exist on both sides of the spacecraft, we here distinguish these elements by the subscripts 1 or 2 .

[26] Equations (1) and (2) indicate that only the bias current inflow is responsible for the total charge variation over each sensing element. On the other hand, since the charge required to maintain the desired $V_{\text {st-s }}$ and $V_{\mathrm{g} \text {-sc }}$ should be provided by electronics installed in the spacecraft body, the variation of the total charge of the spacecraft, the guard, and the stub bodies should be equal to the charge loss due to the bias current provision. It can be easily confirmed by adding equations (1) through (3) that the total charge of the entire spacecraft system is strictly conserved. Note that the charge accumulation due to the plasma impinging and photoelectron emission is taken into consideration in other routines of EMSES than the capacity matrix processing, and the assumption of total charge conservation during the process is sound.

[27] We here introduce the main equation sets used in the capacity matrix processing. The charge variation $\Delta q_{i}$ for the 
$i$ th grid point defined on a certain conducting body surface is given as

$$
\Delta q_{i}=\sum_{j} c_{i j}\left(\phi_{j}-\phi_{j}^{\prime}\right),
$$

where $c_{i j}$ is an element of the capacity matrix precalculated at an initial stage of the simulation and $\phi_{j}$ and $\phi_{j}^{\prime}$ represent the potentials on the $j$ th grid point after and before the potential control (i.e., the capacity matrix processing), respectively. Among these, $\phi_{j}$ is an unknown value and corresponds to one of $\phi_{\mathrm{s} 1}, \phi_{\mathrm{st} 1}, \phi_{\mathrm{g} 1}, \phi_{\mathrm{sc}}, \phi_{\mathrm{g} 2}, \phi_{\mathrm{st} 2}$, and $\phi_{\mathrm{s} 2}$, depending on to which body element the $j$ th grid belongs. Meanwhile, $\phi_{j}^{\prime}$ is a known value. The summation for $j$ should be performed over all grid points defined on the conducting surfaces. In order to compute $\Delta q_{i}$, we must obtain $\phi_{\mathrm{s} 1}, \phi_{\mathrm{sc}}$, and $\phi_{\mathrm{s} 2}$ as $\phi_{j}$ values, which are in the floating condition.

[28] Next, we derive an equation for $\Delta Q$, which is the charge variation on one conducting element. The quantity $\Delta Q$ is obtained by the summation of equation (4) over all grid points defined on the element. For example, the charge variation $\Delta Q_{\mathrm{s} 1}$ for one side of the sensing element is given as

$$
\begin{aligned}
& \Delta Q_{\mathrm{s} 1}=\sum_{i \in G_{\mathrm{s} 1}} \sum_{j} c_{i j}\left(\phi_{j}-\phi_{j}^{\prime}\right) \\
& =\phi_{\mathrm{s} 1} \sum_{i \in G_{\mathrm{s} 1}} \sum_{j \in G_{\mathrm{s} 1}} c_{i j}+\phi_{\mathrm{st} 1} \sum_{i \in G_{\mathrm{s} 1}} \sum_{j \in G_{\mathrm{st} 1}} c_{i j} \\
& +\phi_{\mathrm{g} 1} \sum_{i \in G_{\mathrm{sl}}} \sum_{j \in G_{\mathrm{g} 1}} c_{i j}+\phi_{\mathrm{sc}} \sum_{i \in G_{\mathrm{s} 1}} \sum_{j \in G_{\mathrm{sc}}} c_{i j} \\
& +\phi_{\mathrm{g} 2} \sum_{i \in G_{\mathrm{s} 1}} \sum_{j \in G_{\mathrm{g} 2}} c_{i j}+\phi_{\mathrm{st} 2} \sum_{i \in G_{\mathrm{s} 1}} \sum_{j \in G_{\mathrm{s} 2}} c_{i j} \\
& +\phi_{\mathrm{s} 2} \sum_{i \in G_{\mathrm{s} 1}} \sum_{j \in G_{\mathrm{s} 2}} c_{i j}-\sum_{i \in G_{\mathrm{s} 1}} \sum_{j} c_{i j} \phi_{j}^{\prime} \\
& =\phi_{\mathrm{s} 1} \sum_{i \in G_{\mathrm{s} 1}} \sum_{j \in G_{\mathrm{s} 1 \mathrm{~s} 1}} c_{i j}+V_{\mathrm{st} 1-\mathrm{s} 1} \sum_{i \in G_{\mathrm{s} 1}} \sum_{j \in G_{\mathrm{st1}}} c_{i j} \\
& +V_{\mathrm{g} 1-\mathrm{sc}} \sum_{i \in G_{\mathrm{sl}}} \sum_{j \in G_{\mathrm{gl}}} c_{i j}+\phi_{\mathrm{sc}} \sum_{i \in G_{\mathrm{s} 1}} \sum_{j \in G_{\mathrm{g} 1+\mathrm{sc}+\mathrm{g} 2}} c_{i j} \\
& +V_{\mathrm{g} 2-\mathrm{sc}} \sum_{i \in G_{\mathrm{s} 1}} \sum_{j \in G_{\mathrm{g} 2}} c_{i j}+V_{\mathrm{st2}-\mathrm{s} 2} \sum_{i \in G_{\mathrm{s} 1}} \sum_{j \in G_{\mathrm{s} 2}} c_{i j} \\
& +\phi_{\mathrm{s} 2} \sum_{i \in G_{\mathrm{s} 1}} \sum_{\in \in G_{\mathrm{s} 2}+\mathrm{s} 2} c_{i j}-\sum_{i \in G_{\mathrm{s} 1}} \sum_{j} c_{i j} \phi_{j}^{\prime} \text {, }
\end{aligned}
$$

where $G$ represents a set of grid points composing a certain conducting element, and $G_{\mathrm{x}+\mathrm{y}}$ denotes $G_{\mathrm{x}} \cup G_{\mathrm{y}}$ in our notation. In the derivation of equation (5), $\phi_{j}$ has been replaced by the potentials of the respective conducting elements, and we have used $\phi_{\mathrm{st} 1,2}=\phi_{\mathrm{s} 1,2}+V_{\mathrm{st}-\mathrm{s}}$ and $\phi_{\mathrm{g} 1,2}=\phi_{\mathrm{sc} 1,2}+V_{\mathrm{g} \text {-sc }}$. Since similar equations can be derived also for $\Delta Q_{\mathrm{st}}$, $\Delta Q_{\mathrm{g} 1}, \Delta Q_{\mathrm{sc}}, \Delta Q_{\mathrm{g} 2}, \Delta Q_{\mathrm{st} 2}$, and $\Delta Q_{\mathrm{s} 2}$, seven equations for $\Delta Q$ are obtained in total.

[29] In the set of the seven equations for $\Delta Q$, the potentials $\phi_{\mathrm{s} 1}, \phi_{\mathrm{sc}}$, and $\phi_{\mathrm{s} 2}$ are the unknown quantities. Hence, we use equations (1) through (3) to solve the simultaneous equations for the potentials.

[30] After obtaining all the conducting body potentials, we can calculate $\Delta q_{i}$ by substituting the potentials back into $\phi_{j}$ in equation (4), which directly tells us how to redistribute the surface charge on the instrument. We then compute the modification of an electrostatic field by solving Poisson's equation for the obtained $\Delta q_{i}$. Finally, we add the field

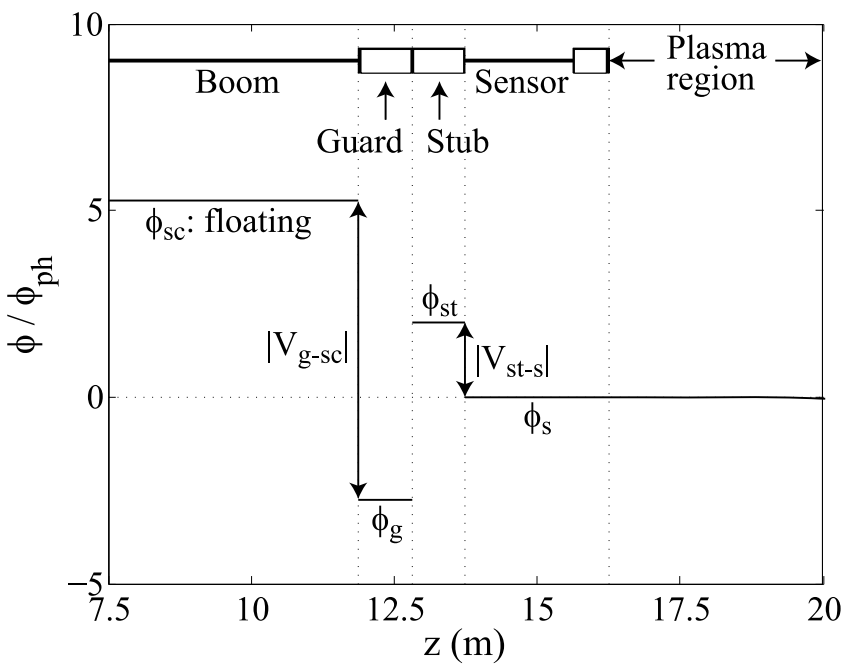

Figure 2. One-dimensional profile of the electric potential along the sensor deployment axis ( $\mathrm{z}$ axis). The horizontal axis value represents the distance from the center of the spacecraft. The profile corresponds to the case of the bias current of $I_{\mathrm{bias}}=-0.75 I_{\mathrm{ph} 0}$, where $I_{\mathrm{ph} 0}$ represents the photoemission current magnitude.

modification to an electric field that has been obtained before the capacity matrix processing.

[31] For verification, we plot a potential pattern measured along the sensor axis penetrating both the conductor and plasma regions obtained from an actual simulation run in Figure 2. The solid line represents the potential profile when $\phi_{\mathrm{s}}$ approaches the background plasma potential $\phi_{\mathrm{bg}}=0$ due to a proper magnitude of $I_{\text {bias }}$. The profile confirms that puck surface locations that correspond to the guard and stub have prescribed potential differences from the boom and sensor bodies, respectively; that is, $\phi_{\mathrm{g}}$ is $8 \phi_{\mathrm{ph}}$ lower than $\phi_{\mathrm{sc}}$, and $\phi_{\mathrm{st}}$ is $2 \phi_{\mathrm{ph}}$ higher than $\phi_{\mathrm{s}}$ correctly. This simulation result verifies the proposed numerical modeling.

\subsection{Advantages and Limitations of the PIC Approach}

[32] The primary objective of the present study is to examine an equilibrium photoelectron distribution around an electric field sensor. Further, in terms of numerical tool development, our ultimate goal is to establish a method with the capability of reproducing the electric field sensor behavior not only in the static condition but also in response to transient processes of the surrounding plasma.

[33] We therefore place emphasis on producing a selfconsistent solution of a system consisting of the plasma particle dynamics and sensor potential variation, which are closely coupled with each other. Actually, the numerical modeling described in section 2.3 enables us to simulate the spacecraft and sensor potentials in the floating potential condition as a whole, while arbitrary potential differences can simultaneously be imposed between specific body elements, e.g., between the guard electrode and the spacecraft body. In principle, this treatment reflects an actual sensor behavior in space environments, which is the major strength of the present approach.

[34] The above self-consistent approach, however, forces us to handle a significant limitation, i.e., poverty of the 
Table 1. System, Background Plasma, and Photoelectron Parameters, Which We Consider in the Present Analysis ${ }^{\mathrm{a}}$

\begin{tabular}{lc}
\hline \multicolumn{1}{c}{ Parameter } & Value \\
\hline System & \\
Grid spacing & $6.3 \times 10^{-1} \mathrm{~m}$ \\
Time step width & $2.4 \times 10^{-1} \mu \mathrm{s}$ \\
System length & $80 \mathrm{~m}$ \\
Background plasma & \\
Number density & $8.5 \mathrm{cc}^{-1}$ \\
Electron and ion temperatures & $10^{5} \mathrm{~K}$ \\
Debye length & $\sim 7.5 \mathrm{~m}$ \\
Electron differential current density & $6.7 \times 10^{-7} \mathrm{Am}^{-2}$ \\
Photoelectron & $1.7 \times 10^{-5} \mathrm{Am}^{-2}$ \\
Current density & $2.2 \mathrm{eV}$ \\
Energy &
\end{tabular}

${ }^{a}$ All simulation parameters are actually determined and given in values normalized to $\Delta r$ and $\Delta t$. Hence, the listed parameter set in physical units is one example converted from such simulation parameters by determining the concrete values of $\Delta r$ and $\Delta t$.

spatial resolution to describe the spacecraft and sensor bodies. The overall sensor dimensions must be enlarged considerably compared with the practical instruments. The deformation is most prominent for the wire radii of the sensing element and boom, and also not negligible for the probe and puck dimensions. Considering the limitations, we must handle simulation outputs with great caution. The enlarged instrument elements greatly exaggerate the photoelectron flux from the instrument bodies and the variation in the potential resulting from using the photoelectron guard electrode. Discussions regarding these important issues will be provided in section 4 .

\subsection{Simulation Setup}

[35] We place the spacecraft body with one pair of the electric field sensors in a three-dimensional simulation box. The sensors are aligned with the $z$ axis, which we define as the sensor axis. The box is then filled with background plasma composed of electrons and protons with finite thermal velocity, the Debye length $\lambda_{D}$ of which is set to $\sim 1 / 10$ times the box size on each side. We then consider a photoemitting situation assuming that the sun illuminates the spacecraft body and the sensors from the $+x$ direction perpendicular to the sensor axis. The boundary conditions for the outer edges of the simulation box are selected so as to realize an isolated system [Miyake et al., 2008]. We use the Neumann condition for the electrostatic potential obtained from Poisson's equation. In order to supplement escaping particles from the simulation box, we inject particles having a velocity distribution given by $F(v)=v f(v)$ from the simulation box edges, where $v$ and $f(v)$ are the initial velocity and the Maxwell velocity distribution, respectively. The particle injection from the outer edge is, of course, performed only for background electrons and ions.

[36] In Table 1, we summarize principal plasma parameters used in the present simulations. Although we constructed the sensor model based on MEFISTO, the objective of the present study is to investigate the general behavior of the modern electric field sensor in a representative plasma condition, rather than to examine a very specific plasma environment in Mercury's magnetosphere. Therefore, in the current analysis, we use one parameter set assuming tenuous plasma environments, which are typically encountered in Earth's magnetosphere and probably also in Mercury's magnetosphere. In such tenuous plasmas, $\lambda_{\mathrm{D}}$ of the background plasma $(10-100 \mathrm{~m})$ is comparable to or larger than the sensor tip-to-tip length (e.g., 30 and $90 \mathrm{~m}$ for MEFISTO and Cluster EFW, respectively). In the current analysis, we use a bit smaller $\lambda_{\mathrm{D}}$ as shown in Table 1 to reduce a necessary size of the simulation box.

[37] The photoelectron is typically denser $\left(10^{3}-10^{5}\right.$ times $)$ and colder $\left(10^{-2}-10^{-1}\right.$ times $)$ than the background plasma. Then, the resultant photoelectron shielding length in the order of $10 \mathrm{~cm}$ is much smaller than both $\lambda_{\mathrm{D}}$ and the sensor tip-to-tip length. Basically, we follow these relations in the present parameter setting. From a more quantitative standpoint, however, the present background plasma setting corresponds to a not so sparse condition, e.g., seen in Earth's magnetosheath, because a too large ratio between the characteristic lengths of the background plasma and the photoelectron is difficult to simulate. We chose the photoelectron temperature $T_{\mathrm{ph}}=1 / 4 T_{\mathrm{e}}$ and flux $\Gamma_{\mathrm{ph}}=25 \Gamma_{\mathrm{e}}$, where $T_{\mathrm{e}}$ and $\Gamma_{\mathrm{e}}$ represent the temperature and differential flux for the background electron, respectively. The settings produce the ratio of the most probable shielding length by photoelectron to $\lambda_{\mathrm{D}}$ being $1 / 14$. Although this value is relatively large compared to those seen in more tenuous magnetospheric plasmas, we can discern principal features of the photoelectron behaviors even in the present simulation setup.

[38] The other principal plasma conditions are determined as follows. We assume an electron-proton background plasma and use the real mass ratio $m_{\mathrm{i}} / m_{\mathrm{e}}=1836$, where $m_{\mathrm{e}}$ and $m_{\mathrm{i}}$ represent the masses of electrons and protons, respectively. The background electron and ion temperatures, $T_{\mathrm{e}}$ and $T_{\mathrm{i}}$, respectively, satisfy an isothermal condition, i.e., $T_{\mathrm{e}}=T_{\mathrm{i}}$. Usually in outer magnetospheric plasmas, the gyroradius of electrons, even for photoelectrons (e.g., $\sim 100 \mathrm{~m}$ for a magnetic field strength $50 \mathrm{nT}$ ), is larger than the sensor tip-to-tip length. Therefore, the effects of the static magnetic field are neglected in the present simulation.

\section{Simulation Results}

\subsection{Bias Current Magnitude}

[39] In order to find a magnitude of $I_{\text {bias }}$ that produces a zero sensor-to-background potential, we run multiple simulations for various $I_{\text {bias }}$ values. We then found that for $I_{\text {bias }}=-0.75 I_{\text {ph0 }}$, the sensor potential successfully approaches the background plasma potential $\phi_{\mathrm{bg}}(=0)$, where $I_{\mathrm{ph} 0}$ represents the photoemission current magnitude. The potential profile along the sensor axis for this $I_{\text {bias }}$ magnitude has been shown in Figure 2. We define this basic case as a reference case and will refer to it for the comparison with other simulations.

[40] For verification, we estimate the resultant value of

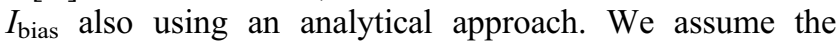
current balance condition is achieved at the sensing element surface among a photoemitting current, impinging background electron and ion currents, and the bias current as follows:

$$
I_{\mathrm{ph} 0}+I_{\text {bias }}-I_{\mathrm{e} 0}+I_{\mathrm{i} 0}=0,
$$



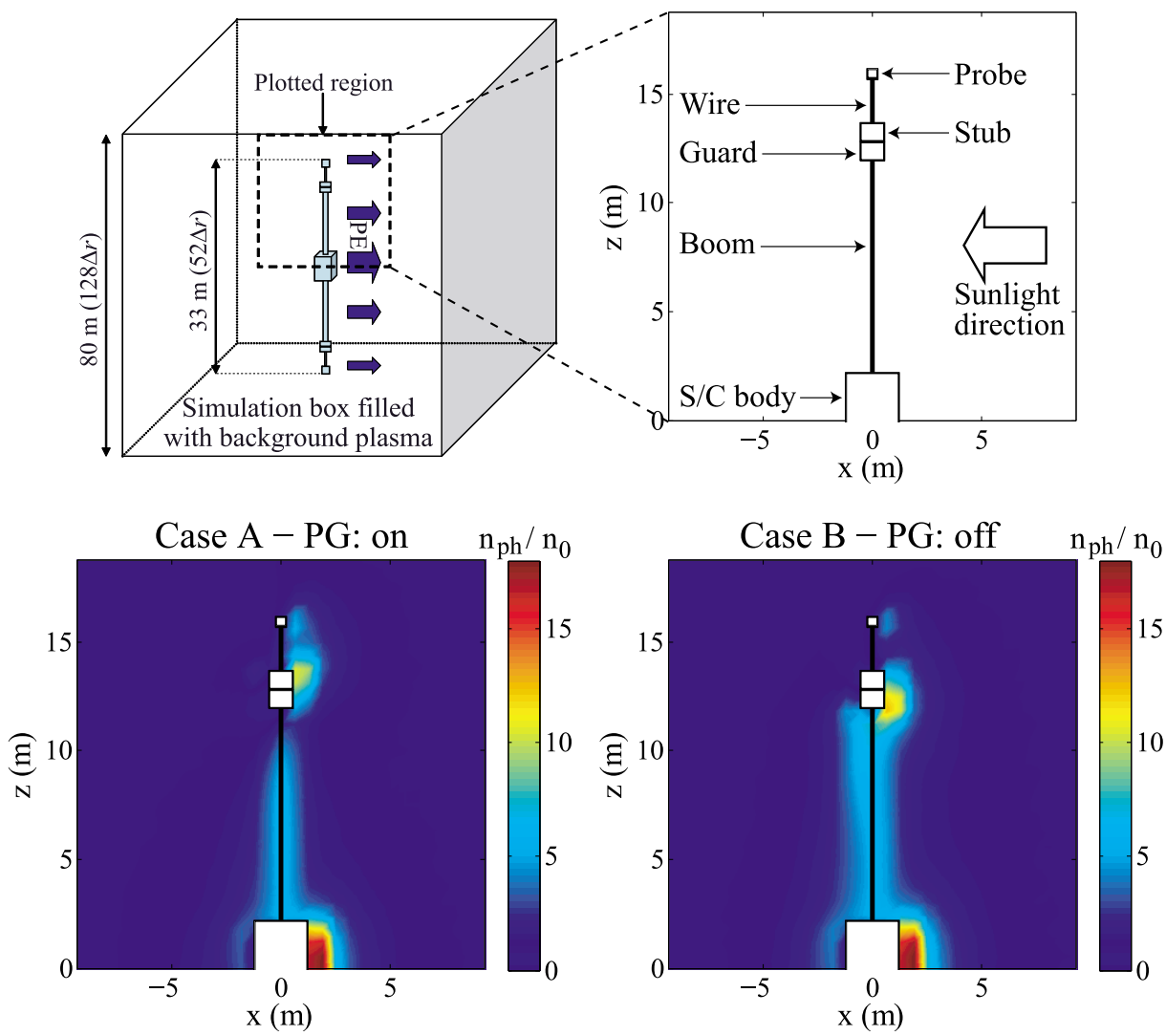

Figure 3. Two-dimensional profiles of the electron density with and without the photoelectron guard (PG) operation on the $x-z$ plane that bisects the center of the spacecraft. (top left) The plotted region in the simulation box. (top right) The sensor and spacecraft positions in the plane. (bottom) Cases of PG: on (case A) and PG: off (case B), respectively. Values are normalized to the background plasma density $n_{0}$.

where $I_{\mathrm{e} 0}$ and $I_{\mathrm{i} 0}$ correspond to the background electron and ion currents, respectively, when $\phi_{\mathrm{s}}=0$. We can derive $I_{\text {bias }}$ from equation (6) as

$$
\begin{aligned}
I_{\text {bias }} & =-I_{\mathrm{ph} 0}+I_{\mathrm{e} 0}-I_{\mathrm{i} 0} \\
& =I_{\mathrm{ph} 0}\left[-1+\frac{S \Gamma_{\mathrm{e}}}{S_{\mathrm{ph}} \Gamma_{\mathrm{ph}}}\left(1-\sqrt{\frac{m_{\mathrm{e}} T_{\mathrm{i}}}{m_{\mathrm{i}} T_{\mathrm{e}}}}\right)\right] \sim-0.79 I_{\mathrm{ph} 0},
\end{aligned}
$$

where $S$ and $S_{\text {ph }}$ represent the total and photoemitting surface areas of the sensing element, respectively. In order to obtain the results, we used the simulation parameters $S \Gamma_{\mathrm{e}} / S_{\mathrm{ph}} \Gamma_{\mathrm{ph}}=0.22$ and $\sqrt{m_{\mathrm{e}} T_{\mathrm{i}} / m_{\mathrm{i}} T_{\mathrm{e}}}=2.33 \times 10^{-2}$. Based on this consideration, the $I_{\text {bias }}$ magnitude resulting from the simulation is found to be $5 \%$ smaller than that obtained by the simplified analytical estimation.

[41] This difference implies the presence of photoelectrons emitted by other conducting bodies and eventually impinging on the sensing element. We tracked the trajectories of all photoelectrons and counted the number of such photoelectrons. As a result, currents with magnitudes of $0.024 I_{\mathrm{ph} 0}, 0.017 I_{\mathrm{ph} 0}$, and $1.3 \times 10^{-3} I_{\mathrm{ph} 0}$ originating from the stub, the guard, and the spacecraft body, respectively, flow into the sensing element. The collection of negative charge due to these currents is concluded to reduce the required magnitude of $I_{\text {bias }}$ in the simulation from the analytical value.
[42] We should note that, in practice, the sensor often aims for an operation not at $\phi_{\mathrm{s}} \sim 0$ but at $\phi_{\mathrm{s}}$ being a somewhat positive value. Such an operation can prevent $\phi_{\mathrm{s}}$ from falling into an unstable negative potential range even in cases of an anomalistic increase of background electron impinging and an anomalistic decrease of photoelectron emission. Thus, the $I_{\text {bias }}$ magnitude is practically chosen to include a certain level of safety margin. Although we basically deal with the $\phi_{\mathrm{s}} \sim 0$ condition in the following analysis to study the sensor being close to the background potential conceptually, we will also consider an influence of the $I_{\text {bias }}$ margin in section 4.3 .

\subsection{Photoelectron Density Around the Sensor}

[43] Our next interest is in the equilibrium photoelectron distribution around the sensor and the spacecraft. Figure 3 shows color maps of the electron density at the steady state. Figure 3 (bottom left) corresponds to the reference case. Figure 3 (bottom right) shows the simulation result for the case in which the photoelectron guard is disabled; that is, we set both $V_{\text {st-s }}$ and $V_{\mathrm{g} \text {-sc }}$ to zero. Note that the current biasing operation is performed both in cases A and B.

[44] In both cases, we confirmed electron dense regions primarily on the sunlit side of each conducting body. The distribution of the photoelectron clouds, however, differs greatly between the two cases, particularly in the vicinity of 

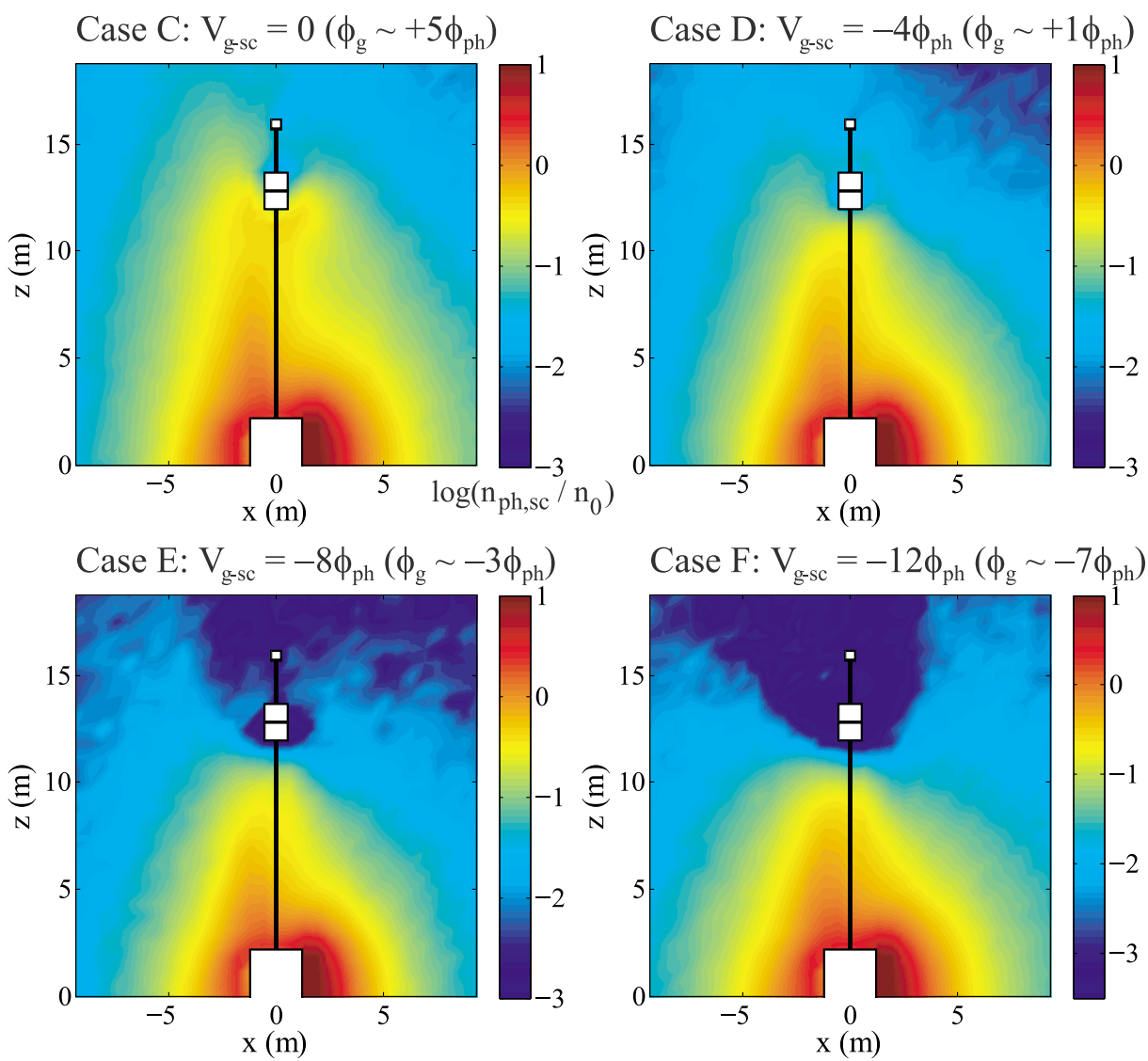

Figure 4. Two-dimensional density profiles of photoelectrons emitted from the spacecraft body measured on a logarithmic scale for case $\mathrm{C}\left(V_{\mathrm{g} \text {-sc }}=0\right)$, case $\mathrm{D}\left(V_{\mathrm{g} \text {-sc }}=-4 \phi_{\mathrm{ph}}\right)$, case $\mathrm{E}\left(V_{\mathrm{g} \text {-sc }}=-8 \phi_{\mathrm{ph}}\right)$, and case $\mathrm{F}\left(V_{\mathrm{g}-\mathrm{sc}}=-12 \phi_{\mathrm{ph}}\right)$. Values are normalized to the background plasma density $n_{0}$.

the sensor and the puck surfaces. Comparison of cases A and $\mathrm{B}$ reveals the effects of puck surface potential control on the density distributions around the guard and stub surfaces. In case A, the photoelectron cloud is created primarily on the stub surface, whereas, in case B, the cloud shifts its position onto the guard surface. As a result, the cloud on the puck coalesces with that surrounding the boom in case B.

[45] The results are understandable by the consideration of the potential relation between the guard and the stub, because the photoelectron cloud tends to be formed on the surface at a higher electric potential. In case $\mathrm{A}, \phi_{\mathrm{st}}$ is higher than $\phi_{\mathrm{g}}$ as shown in Figure 2, whereas, in case B, this potential relation is reversed, because $\phi_{\mathrm{st}}$ and $\phi_{\mathrm{g}}$ are identical to $\phi_{\mathrm{s}} \sim 0$ and $\phi_{\mathrm{sc}} \sim+5 \phi_{\mathrm{ph}}$, respectively.

[46] A difference between the two cases is also seen around the boom. For case A, the electron density evidently decreases near the guard. This tendency is due to an effect of the negatively biased potential of the guard with respect to the boom. Conversely, in case B, the electron density around the boom becomes higher near the guard.

[47] The simulation results qualitatively clarified how the photoelectron distribution is influenced by the photoelectron guard operation at the sensor, the puck, and the boom. However, in practice, the photoelectron density around the sensor, puck, and boom should be much smaller than is demonstrated here, because a practical instrument generally has much smaller dimensions than the spacecraft body than is represented by the present model. Therefore the spacecraft-originating photoelectron distribution deserves intensive study before any other photoelectron components. In sections 3.3 and 3.4, we consider how the spacecraftoriginating photoelectron distribution is influenced by the guard electrode operation.

\subsection{Distribution of Spacecraft-Originating Photoelectrons}

[48] The primary objective of the guard electrode is to exclude photoelectrons emitted by the spacecraft body. We here concentrate on the distribution of the spacecraftoriginating photoelectrons around the sensor. For a detailed analysis, we have newly performed two simulations for the parameters of $V_{\mathrm{g}-\mathrm{sc}}=-4 \phi_{\mathrm{ph}}$ and $-12 \phi_{\mathrm{ph}}$ in addition to the reference case. For the stub potential magnitude, we follow the relation of $\left|V_{\text {st-s }}\right| /\left|V_{\mathrm{g} \text {-sc }}\right|=1 / 4$ as used in the reference case. We also adjusted the $I_{\text {bias }}$ magnitude so that $\phi_{\mathrm{s}}$ approaches $\phi_{\mathrm{bg}}$.

[49] Figure 4 shows the two-dimensional density profiles of only photoelectrons emitted from the spacecraft body measured on a logarithmic scale for case C: $V_{\mathrm{g} \text {-sc }}=0$, case $\mathrm{D}$ : $-4 \phi_{\mathrm{ph}}$, case E: $-8 \phi_{\mathrm{ph}}$, and case F: $-12 \phi_{\mathrm{ph}}$. Note that cases C and $\mathrm{E}$ (Figure 4) are the same simulations as cases B and A (Figure 3), respectively, in section 3.2.

[50] As an overall trend, it is confirmed in all cases that the photoelectron density decreases rapidly with increasing 


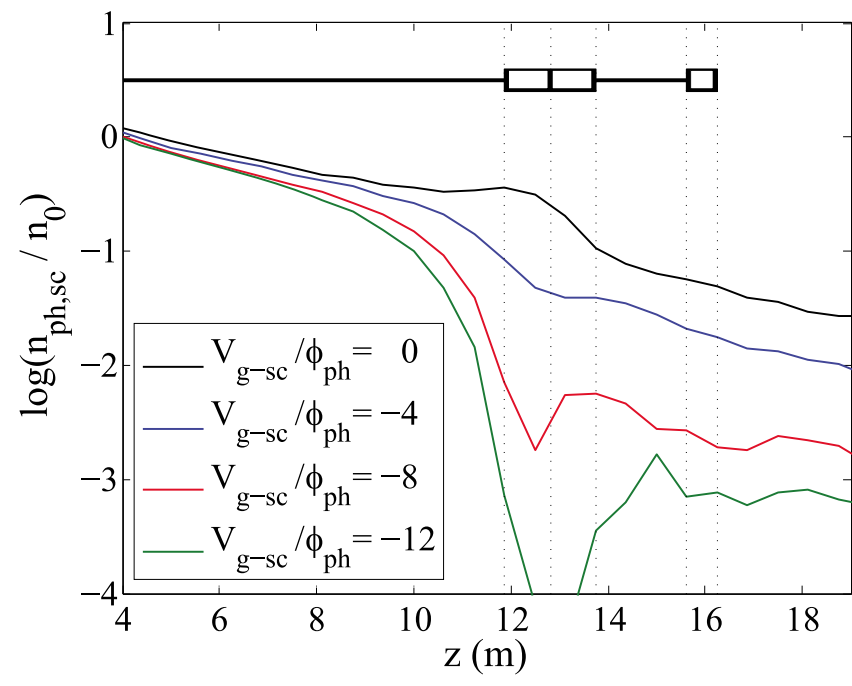

Figure 5. One-dimensional density profiles of the spacecraft-originating photoelectrons measured along an axis $(x, y)=(-1.25 \mathrm{~m}, 0)$ aligned in the $z$ direction and passing through a location adjacent to the sensor.

distance from the spacecraft body. The photoelectron cloud is also observed to extend most distantly in the sensor axis direction due to the presence of a potential well for electrons around the positively charged boom conductor.

[51] Figure 4 (top left) corresponds to the case that the photoelectron guard electrode is not operated. Even in this guard-disabled case, the photoelectron density along the sensor axis decreases at the interface between the guard and the stub, i.e., at $z \sim 13 \mathrm{~m}$. In this case, $\phi_{\mathrm{st}}$ is $\sim 5 \phi_{\mathrm{ph}}$ lower than $\phi_{\mathrm{g}}$ as described in section 3.2. This difference in potentials causes a local decrease in the photoelectron density at the interface. Nevertheless, a photoelectron cloud of density $\sim 0.05 n_{0}$ approaches the probe position from the sunless $(-x)$ side, where $n_{0}$ represents the background plasma density.

[52] For cases D-F, i.e., the cases in which the photoelectron guard electrode is operated, the photoelectron density is decreased at the guard position $(z \sim 12 \mathrm{~m})$ as compared to case $\mathrm{C}$. The density decrease is then more prominent for larger magnitudes of $V_{\mathrm{g} \text {-sc }}$. This result clearly reflects the benefit associated with the use of the guard electrode.

[53] Although we so far give $V_{\mathrm{g} \text {-sc }}$ values as the guard potential parameter of the simulations, $\phi_{\mathrm{g}}$ as measured by referencing the background plasma potential $\phi_{\text {bg }}$ can also be an important factor. In the present simulation, case D corresponds to $\phi_{\mathrm{g}}>\phi_{\mathrm{bg}}$, whereas cases $\mathrm{E}$ and $\mathrm{F}$ correspond to $\phi_{\mathrm{g}}<\phi_{\text {bg. }}$. The remarkable feature is that, unlike in case D, in cases $\mathrm{E}$ and $\mathrm{F}$ the photoelectron sparse region with a bowlike boundary clearly spreads for $z>12 \mathrm{~m}$. The photoelectron sparse region encompasses the probe position in cases $\mathrm{E}$ and $\mathrm{F}$.

[54] We also observe this signature with the onedimensional density profile shown in Figure 5, which is measured along the sensor deployment direction at a location $(x, y)=(-1.25 \mathrm{~m}, 0)$ adjacent to the sensor. A notable difference in density signatures is observed at $z>11 \mathrm{~m}$. In case $\mathrm{D}\left(V_{\mathrm{g}-\mathrm{sc}}=-4 \phi_{\mathrm{ph}}\right)$, the logarithmic curve of the density decreases at a nearly constant rate, except that a local dip in density is observed near the guard position. In contrast, in cases $\mathrm{E}\left(V_{\mathrm{g} \text {-sc }}=-8 \phi_{\mathrm{ph}}\right)$ and $\mathrm{F}\left(V_{\mathrm{g} \text {-sc }}=-12 \phi_{\mathrm{ph}}\right)$, a drastic decrease in density is clearly observed at the guard position, and considerably low density is maintained also at $z>13 \mathrm{~m}$. Eventually, the photoelectron density decreases to $10^{-2.5} n_{0}$ and $10^{-3} n_{0}$ at the probe position in cases $E$ and F, respectively. Implications of these resultant densities will be discussed in section 4.1 .

\subsection{Effects of Photoelectron Guard Electrode}

[55] In the series of simulations $C$ through $F$, we increased $\left|V_{\mathrm{g} \text {-sc }}\right|$ by $4 \phi_{\mathrm{ph}}$ increments. Nevertheless, the photoelectron density at the probe position shown in Figure 5 decreased most prominently between cases $\mathrm{D}$ and $\mathrm{E}$, at which the guard potential condition transitions from $\phi_{\mathrm{g}}>\phi_{\mathrm{bg}}$ to $\phi_{\mathrm{g}}<$ $\phi_{\text {bg. }}$. If most photoelectrons move just along the boom conductor, this result is difficult to interpret, because the orbits of such photoelectrons should have little relevance to the difference between $\phi_{\mathrm{g}}$ and $\phi_{\mathrm{bg}}$.

[56] In order to resolve the difference between the cases of $\phi_{\mathrm{g}}>\phi_{\mathrm{bg}}$ and $\phi_{\mathrm{g}}<\phi_{\mathrm{bg}}$, we show the photoelectron flow around the sensor for cases $\mathrm{D}$ and $\mathrm{F}$ as vector plots in Figure 6. In Figure 6, the background faint color maps represent the photoelectron density and are identical to Figure 4.

[57] The profiles clearly show the leftward photoelectron flow at the sunlit side of the instrument in both cases. Meanwhile, it is difficult to observe a photoelectron flow along the boom conductor except at a very limited region in case D, e.g., at the nearest neighbor of the boom conductor at $z \sim 9 \mathrm{~m}$. In case $\mathrm{D}$, the photoelectrons approaching from right eventually arrive at the sunlit side of the puck and sensing element surfaces. In case $\mathrm{F}$, the guard electrode strongly deflects the photoelectrons and prevents them from approaching the sensing element position.

[58] Next, in Figure 7, we show the contour lines of the electric potential around the sensor. Figure 7 (top) shows the overall potential profiles in the same scale as Figure 4, while Figure 7 (bottom) shows the close-up view for the vicinity of the puck. In Figure 7 (top), the background faint color maps show the photoelectron density. Figure 7 (top) clearly shows that the remarkable potential difference between cases $\mathrm{D}$ and $\mathrm{F}$ is localized in the vicinity of the puck, although the photoelectron density difference is distributed more broadly for $z>12 \mathrm{~m}$. These profiles suggest that the local potential structure created by the puck influences the photoelectron density in the broader area than the potential structure itself.

[59] As shown in the close-up view of case $F$, a potential hump for photoelectrons (i.e., a negative potential region) is formed at the right and left sides of the guard position, whereas a potential well (i.e., a positive potential region) is formed at the sides of the stub position. The potential hump is of particular importance for repelling approaching photoelectrons. We confirm that a similar, but smaller-scale potential hump is also formed in case $\mathrm{E}$ (not shown). In case $\mathrm{D}$, on the other hand, such a hump, even a small one, does not appear around the puck as shown in Figure 7 because the guard itself has a positive potential with respect to $\phi_{\mathrm{bg}}$.

[60] In Figure 8, we summarize the potential structure around the sensor and its effect on the flow of photoelec- 

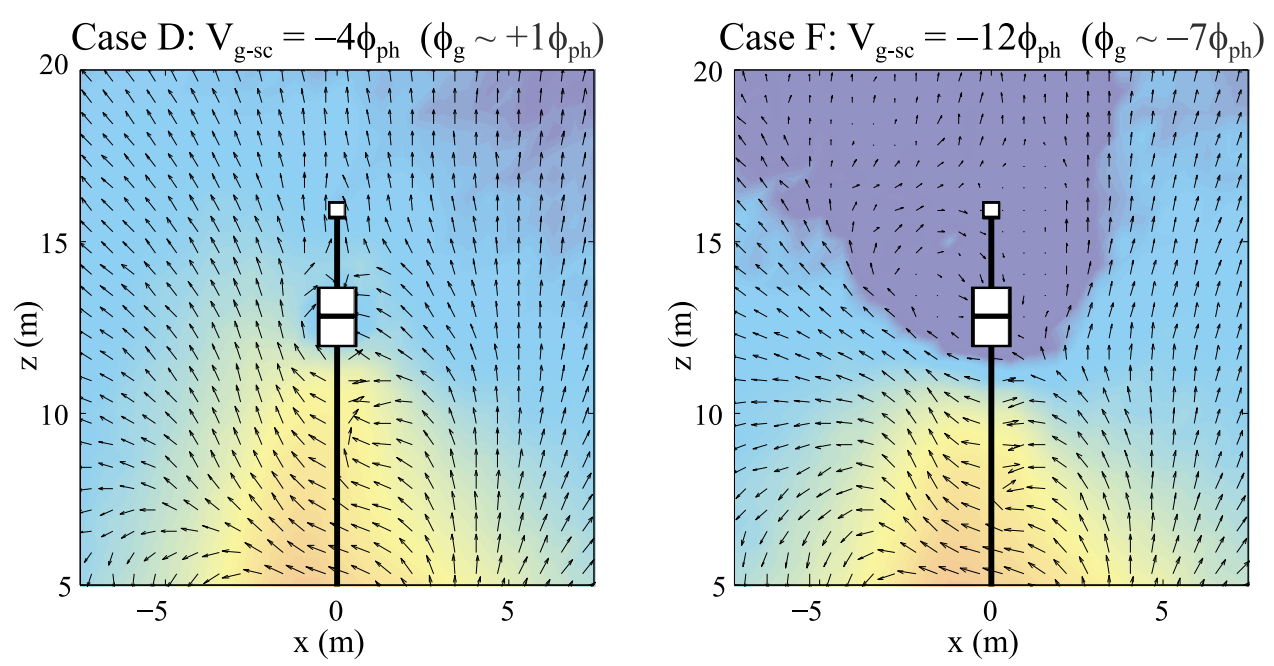

Figure 6. Vector plots of the photoelectron flow for case D $\left(V_{\mathrm{g}-\mathrm{sc}}=-4 \phi_{\mathrm{ph}}\right)$ and case $\mathrm{F}\left(V_{\mathrm{g}-\mathrm{sc}}=-12 \phi_{\mathrm{ph}}\right)$. The background color maps show the spacecraft-originating photoelectron density (same as Figure 4).
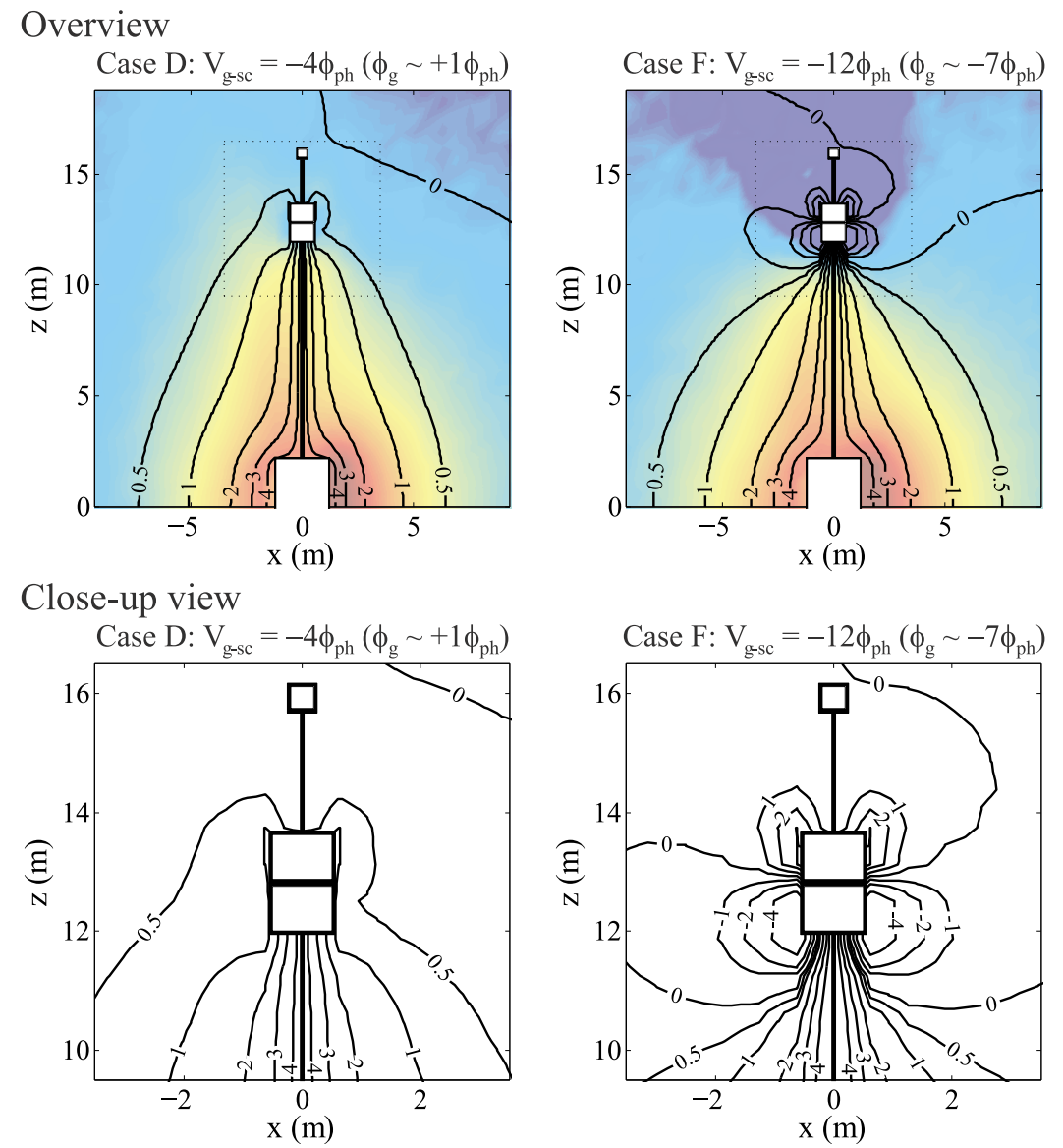

Figure 7. Contour line plots of the electric potential for case $\mathrm{D}\left(V_{\mathrm{g}-\mathrm{sc}}=-4 \phi_{\mathrm{ph}}\right)$ and case $\mathrm{F}\left(V_{\mathrm{g}-\mathrm{sc}}=-12 \phi_{\mathrm{ph}}\right)$. (top) An overview and (bottom) a close-up view for the vicinity of the puck, respectively. Figure 7 (bottom) focuses on the region that is indicated by dashed line boxes in Figure 7 (top). Potential values are normalized to $\phi_{\mathrm{ph}}$. 

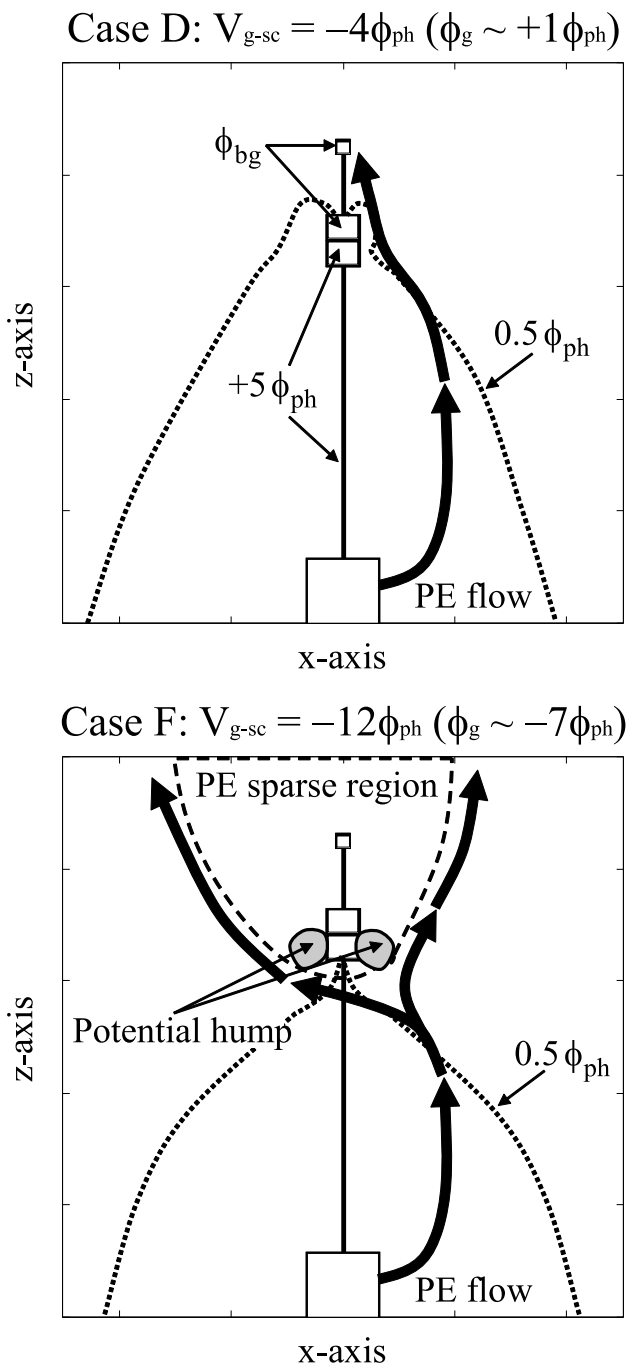

Figure 8. Schematic illustrations of the potential structures influenced by the photoelectron guard electrode and the photoelectron (PE) flow approaching the puck and sensor locations. The dotted lines represent contour lines of potential. The dashed line in case $\mathrm{F}$ indicates a photoelectron sparse region created by the photoelectron guard operation.

trons approaching the puck and sensing element locations as schematic illustrations. In both cases, there are a substantial number of photoelectrons that do not flow along the boom conductor but take a path shown in Figure 8. The direction of the photoelectron flow gradually tilts to the left as the photoelectrons move away from the spacecraft body, because the potential well surrounding the boom conductor tapers toward the boom tip.

[61] In case D, the approaching photoelectrons cannot be effectively deflected by the guard electrode due to the absence of the potential hump. Consequently, these photoelectrons tend to approach the sunlit side of the puck and sensing element surfaces. Although the difference in potential between the boom and guard bodies would be able to repel photoelectrons if they flow along the boom conductor, such photoelectrons are actually in the minority in the present simulations, which leads to the insufficient guard effects observed in case D.

[62] On the other hand, for case $\mathrm{F}$, the photoelectrons approaching the potential hump are deflected in the direction away from the guard electrode. Then, the bow-like signature of the photoelectron sparse region is formed along the flow direction of the deflected photoelectrons. The remarkable feature is that the flow curvature of the deflected photoelectrons is too small to move behind the potential hump, at which the stub and the sensing element are located. As a result, the photoelectron sparse region has a much larger scale than the potential hump itself enough to encompass the probe position.

[63] The above process can effectively prevent spacecraftoriginating photoelectrons from arriving at the sensing element location, although the potential hump does not cover the probe position by itself. Hence, the formation of the potential hump, particularly at the sunlit side of the puck, is a rather significant than merely differentiating $\phi_{\mathrm{g}}$ from $\phi_{\mathrm{sc}}$. This lesson provides us an important guideline on the guard operation that $\phi_{\mathrm{g}}$ should be lower than not only $\phi_{\mathrm{sc}}$ but also $\phi_{\mathrm{bg}}$ in order to create the potential hump.

[64] The effective area of the potential hump also depends on the puck dimensions as well as $\phi_{\mathrm{g}}$. We additionally performed two simulations to examine the dependence of the guard effect on the puck dimensions, in which we doubled the puck size in the $x$ or $z$ directions from the original model. We primarily confirmed in both two cases that the photoelectron sparse region becomes greater than in the case of the original model. We then found that the photoelectron sparse region is greater for the puck that is longer in the $x$ direction than for the puck that is longer in the $z$ direction. This difference reveals the importance of a potential hump expansion in the radial direction to effectively repel photoelectrons coming from the sun direction. This result may be important for considering an optimum configuration of the guard electrode.

\section{Discussions}

\subsection{Practical Implications of the Guard Operation}

[65] The present study proposes a new guideline on the operational conditions of the guard electrode, specifically regarding the guard potential setting. The guard operation within the guideline achieves a more effective reduction of the spacecraft-originating photoelectrons at the sensing element position. We here discuss the practical implications of such a guard operation in the use of the electric field sensor in space.

[66] The merit of excluding photoelectrons from the spacecraft body is accounted for several reasons. The spacecraft-originating photoelectrons can influence important electric properties such as a contact resistance between the sensor and the background plasma. Because the spacecraft-originating photoelectron influx is generally not easy to quantify at the site, there is a merit to minimize the photoelectron inflow to the sensor, from the perspective that we should know the basic sensor properties precisely. Furthermore, when the spin axis is tilted to the sunlight direction unlike the present situation, the spacecraft-originating photoelectrons would cause different floating potentials between one pair of probes depending on whether each 
probe is directed sunward or antisunward. The unbalanced charging between the probes is measured as if there is a spurious electric field. This effect should be minimized by preventing the photoelectrons from approaching the probe position.

[67] Taking the above reasons into consideration, making the spacecraft-originating photoelectron density sufficiently less than the background plasma density is a reasonable target of the guard operation. Except for the bias current, the background plasma electron current is typically a major counterpart to the photoemission current in achieving the current balance at the sensor. Hence, the spacecraftoriginating photoelectron influx would have little influence if the photoelectron density is comparable to or less than the background plasma density, because the photoelectron thermal velocity is much smaller than that of the background electron.

[68] In this sense, the guard operations performed in cases $\mathrm{E}$ and $\mathrm{F}$ of section 3.3 demonstrated a sufficient performance. In these cases, the photoelectron densities at the sensor position are $0.03 \mathrm{cc}^{-1}$ and $0.009 \mathrm{cc}^{-1}$, respectively, where we use $n_{0} \sim 8.5 \mathrm{cc}^{-1}$ listed in Table 1 . These density values are comparable to the most tenuous plasmas encountered in the Earth's magnetosphere, and thus the spacecraft-originating photoelectron influx would have very limited influence on the sensor potentials even in the tenuous plasma environment.

[69] Note that even in cases $\mathrm{C}$ and $\mathrm{D}$ of section 3.3, the photoelectron density is smaller than the background plasma density at the sensing element position as shown in Figure 5. This is because of our parameter choice assuming a not so sparse background plasma. In the physical unit, the photoelectron density at the sensor position is $0.14 \mathrm{cc}^{-1}$ in case D. In practice, we can consider much more tenuous magnetospheric plasmas than this value: e.g., $\sim 10^{-2} \mathrm{cc}^{-1}$ typically seen in Earth's magnetotail lobes. In such an environment, the spacecraft-originating photoelectrons of the density $0.14 \mathrm{cc}^{-1}$ would be problematic in influencing the current balance formed at the sensing element. More precisely, we should consider a possibility that the profile in Figure 5 would change slightly depending on the background plasma density. However, this brief discussion reconfirms the significance of the proposed guideline for optimal guard operations.

[70] We finally mention about a guard effect on photoelectrons emitted by the puck itself. In contrast to the clear merit on the spacecraft-originating photoelectrons, it is confirmed in the simulations that the guard operation is not so effective to reduce the puck-originating photoelectron inflow to the sensor. This might be a problem in the use of the electric field sensor. Actually we have shown in the bias current calculation described in section 3.1 that the puckoriginating photoelectron inflow is a major reason for the difference between the simulation and the analytical estimation. Although this result is definitely much exaggerated due to the considerably larger puck used in the present simulations than in practice, more quantitative estimation for the puck-originating photoelectron effect would be required as a next step of the research by performing additional simulations focusing more on the region around the puck and the sensor.

\subsection{Considerations for the Realistic Guard Electrode Size}

[71] We next discuss an important issue coming from the present instrument size, which differs from that in practice.

[72] The unrealistically large puck used in the present simulation exaggerates the variation in the potential resulting from using the photoelectron guard electrode. The key point of our simulation result is that the guard electrode should create a potential hump in order to effectively repel spacecraft-originating photoelectrons. Such a potential hump will be created even for electrodes that are much smaller than the present model, provided the guard itself has a negative potential, because the potential hump is centered on the guard. However, the hump will obviously become small for such small electrodes, which would influence the guard utility. For a rough estimation of the practical hump size, we must simplify the hump structure (e.g., a potential created by a spherical conductor) and also neglect potential influences from other instrument elements and the Debye shielding effect. In the case of a spherical guard electrode having a constant potential, the size of a created potential hump should be simply proportional to the electrode radius. Based on this consideration, the hump size $\sim 2 \mathrm{~m}$ obtained in case $\mathrm{F}$ of the present analysis would be decreased to $\sim 10 \mathrm{~cm}$ for the practical guard dimension of a few $\mathrm{cm}$, where we define the hump size as a distance with which the negative potential created by the guard electrode decays to $-\phi_{\mathrm{ph}}$.

[73] If the hump radius is reduced from $2 \mathrm{~m}$ to $10 \mathrm{~cm}$, the cross section of the hump becomes $1 / 400$. This appears to reduce substantially the degree of the photoelectron flow deflection by the guard electrode. However, we can also consider other factors conversely increasing the degree of the photoelectron flow deflection in practice. One of such factors is the size of the potential well created by the positively charged boom conductor. In this paper, we have shown that the spacecraft-originating photoelectrons approach the sensing element position approximately along the boundary of the potential well created around the boom (see Figure 8). Because the radius of the boom conductor $\sim 6 \mathrm{~cm}$ is greatly exaggerated in the present model compared with $0.1-1 \mathrm{~mm}$ in practice, the potential well around the boom would actually be much smaller. This means that the photoelectron flow should be more concentrated and condensed near the boom, which tends to increase the fraction of the photoelectron flow influenced by even the small potential hump.

[74] The actual degree of the photoelectron flow deflection would be determined by a mixture of multiple factors such as described above. Actually, it is not easy to answer to this problem definitely by means of an analytical approach. Eventually, we require a further simulation analysis, in which we can model the guard electrode size more realistically. This important issue should be tackled after the improvement of the spatial resolution of our approach as the future work.

\subsection{Practical Setting of Bias Current Magnitude}

[75] In section 3.1, we calculated $I_{\text {bias }}$ based on the policy of zeroing the sensor-to-plasma potential. This policy is optimal only in a conceptual sense, and such a large bias current will not be used in practice. The reason is that the 
sensor potential at that operating point is too susceptible to even small perturbation of surrounding plasma conditions. Instead, $I_{\text {bias }}$ is usually chosen to include a certain level of margin to avoid such susceptibility. We here consider the guard performance in case of such a practical $I_{\text {bias }}$ setting.

[76] We perform additional simulations for the parameter of $I_{\text {bias }}=-0.7 I_{\text {ph0 }}$, which corresponds to $\sim 0.9$ times the $I_{\text {bias }}$ magnitude used in section 3 . Other simulation settings are common to the simulations described in section 3. In this case, we obtain an equilibrium sensor-to-background potential $\phi_{\mathrm{s}} \sim+0.64 \phi_{\mathrm{ph}}$ as a simulation result.

[77] We then examine an equilibrium photoelectron distribution. In summary of the result, the guard effect of repelling spacecraft-originating photoelectrons is maintained also in case with the $I_{\text {bias }}$ margin, although an increase in photoelectron density is to some degree inevitable at the sensing element position. In Figure 9, we show the influence of the $I_{\text {bias }}$ margin on the one-dimensional density profiles in case $\mathrm{D}\left(V_{\mathrm{g}-\mathrm{sc}}=-4 \phi_{\mathrm{ph}}\right)$ and case $\mathrm{F}\left(-12 \phi_{\mathrm{ph}}\right)$. As shown in Figure 9 , the $I_{\text {bias }}$ margin mainly affects the photoelectron density for $z>13 \mathrm{~m}$. Although the density at the guard position is increased prominently in case $\mathrm{F}$, the density at the probe position is increased moderately only by a factor of $\sim 1.7$. Next, in Figure 10, we show the photoelectron density and flow profiles for case F. We can observe the photoelectron deflection resulting from using the guard electrode as in the case without the $I_{\text {bias }}$ margin. Meanwhile, we also confirm that a larger fraction of the deflected photoelectrons eventually comes in the photoelectron sparse region in comparison with Figure 6 (case F). This photoelectron intrusion is caused by the sensor and stub bodies, which are charged more positively than the case without the $I_{\text {bias }}$ margin.

[78] In this section, we study an effect of the $I_{\text {bias }}$ margin practically introduced in order to increase the stability of the sensor performance against an operating point perturbation. The additional simulations confirm the validity of the guard

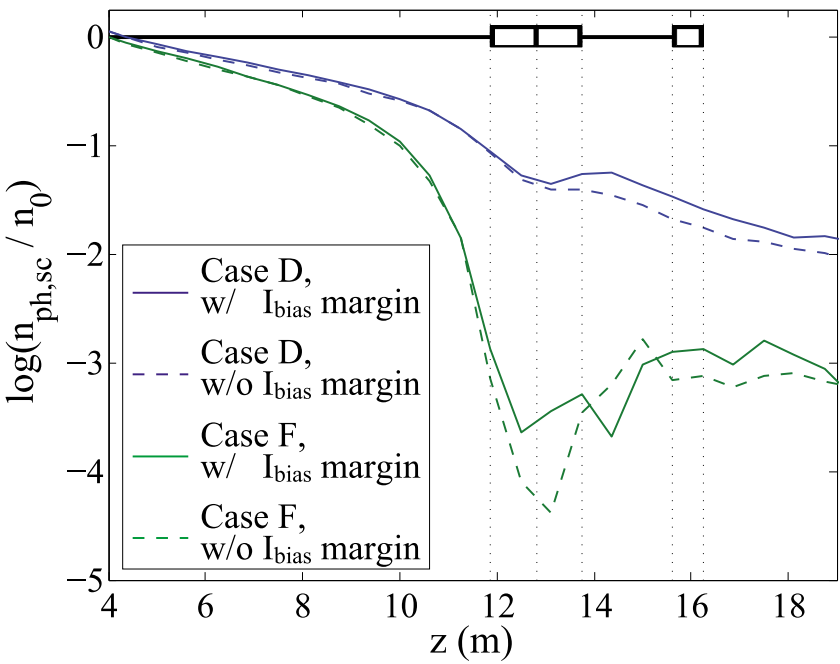

Figure 9. Comparison of one-dimensional profiles of spacecraft-originating photoelectron density between cases with (w/) and without (w/o) the $I_{\text {bias }}$ margin. The blue and green lines correspond to case $\mathrm{D}\left(V_{\mathrm{g} \text {-sc }}=-4 \phi_{\mathrm{ph}}\right)$ and case $\mathrm{F}\left(V_{\mathrm{g}-\mathrm{sc}}=-12 \phi_{\mathrm{ph}}\right)$, respectively.

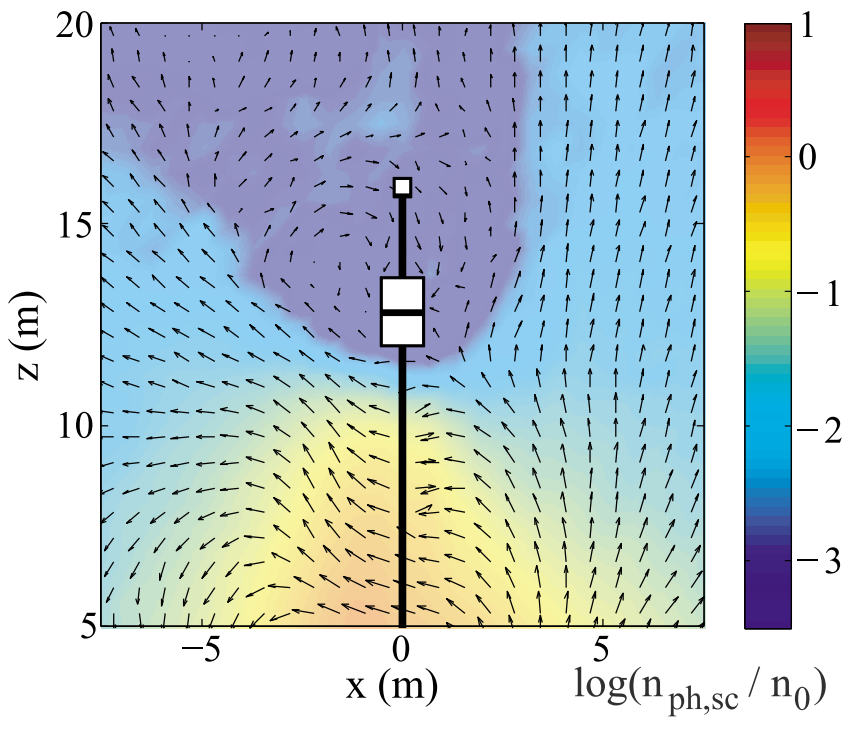

Figure 10. Spacecraft-originating photoelectron flow plotted on a faint color map of the photoelectron density for case $\mathrm{F}\left(V_{\mathrm{g}-\mathrm{sc}}=-12 \phi_{\mathrm{ph}}\right)$ with the $I_{\mathrm{bias}}$ margin.

electrode effect also in case that $\sim 10 \%$ of $I_{\text {bias }}$ used in section 3 is preserved as a margin. Meanwhile, the simulation result also suggests that a too large margin would reduce the effect of the photoelectron guard electrode, which is in a trade-off relation with the assured stability of the sensor potential.

\section{Conclusion}

[79] We have developed a numerical model of a doubleprobe electric field sensor for EMSES, which is the selfconsistent full particle simulation. The proposed model includes the photoelectron guard electrode and current biasing, both of which are key concepts in ensuring an optimum plasma environment around the electric field sensor. These functions are realized in the simulation as a potential control implemented as the extension of the conventional capacity matrix technique, consistently assuring conservation of the total charge of the entire spacecraft and instrument surfaces.

[80] The proposed model is applied to an analysis of the equilibrium plasma environment around the sensor in a photoemitting situation. The present study focuses on the effect of the photoelectron guard electrode on the spacecraft-originating photoelectron distribution. In case of the sun illuminating the spacecraft body from the direction perpendicular to the sensor axis, a portion of the photoelectrons emitted from the spacecraft arrives at the sunward side of the puck and sensing element surfaces. Such a photoelectron flow is largely deflected only when the photoelectron guard electrode creates a negative potential hump at the side.

[81] The simulation results draw out a significant guideline on the guard operation in the use of the electric field sensor; the guard potential should be not only lower than the spacecraft potential but also lower than the background plasma potential. Such a setting causes the photoelectron 
sparse region to become larger than the size of the potential hump, which effectively enfolds the probe position. The proposed guideline also provides us a notable lesson in determining a magnitude of the guard-to-spacecraft potential, because the necessary magnitude generally depends on the surrounding plasma conditions. For example, the required magnitude of the guard-to-spacecraft potential would become up to several $10 \mathrm{~V}$ for the case of very tenuous plasmas, such as those in the lobe region, in which the spacecraft potential can reach up to that magnitude. A simple solution to this problem is to determine a fixed value of the guard potential referenced to a sensor potential rather than to the spacecraft potential, as is performed in Cluster EFW [Gustafsson et al., 1997].

[82] As mentioned in section 2, some parts of the present sensor model are largely deformed from the practical instrument, which introduces some difficulty in extracting quantitative information from the simulation. The effective area of the photoemitting surfaces of the puck, boom, and sensing element is large, and the density ratio between the photoelectrons and the background plasma is small compared to that of a typical tenuous plasma environment. Furthermore, we should also consider another factor resulting from the assumption of a single Maxwellian photoelectron energy distribution, unlike a realistic distribution composed of multiple energy components [Scudder et al., 2000]. Practically, a higher-temperature component may increase the proportion of photoelectrons arriving at the sensing element.

[83] All of the above factors enhance the importance of the guard electrode performance with respect to the spacecraft-originating photoelectrons demonstrated in the present study. Further improvement of the sensor structure modeling is required in the self-consistent PIC simulation for the quantitative evaluation of the electric coupling among multiple conducting elements due to photoelectron currents. Such a quantitative evaluation should be tackled by applying more sophisticated numerical algorithms and largerscale supercomputing techniques, such as a local mesh refinement algorithm and a load-balancing technique for a massively parallel computation [e.g., Nakashima et al., 2009], respectively. We can also use some quasi-analytical models for describing local field variation around the extremely small sensor structure. If the application of such analytical models is limited to the very vicinity of the sensor structure, the plasma dynamics emerging at larger scales can be processed in a self-consistent manner as is conventionally done. We should consider such an approach in the next stage of the research.

[84] Several issues remain as possible targets of an analysis using the present PIC model. For an oblique incident angle of the sunlight, unlike in the present study, the spacecraft-originating photoelectrons are emitted in an asymmetrical manner. This asymmetry may cause a severe imbalance in electric properties between a pair of sensors deployed oppositely with each other and the generation of a spurious electric field. This issue should also be examined in a future numerical study. Another area for future consideration is the nonstationary behavior of electric field sensors, such as those used in the measurement of oscillating fields, which is also an important function of double-probe instruments. We believe that the approach of the present study has significant advantages for such a problem in that this approach can resolve the plasma dynamics, field evolution, and transient process of the instrument charging in a self-consistent manner.

[85] Acknowledgments. The authors would like to thank L. Åhlén for his helpful comments on the proposed numerical model of the instrument and Y. Omura for his comments on the plasma process around the instrument. The computations in the present study were performed using the KDK system of Research Institute for Sustainable Humanosphere (RISH) and the Fujitsu HX600 Cluster of Academic Center for Computing and Media Studies (ACCMS) at Kyoto University. The present study was supported in part by a Grant-in-Aid for Research Fellows from the Japan Society for the Promotion of Science (JSPS).

[86] Masaki Fujimoto thanks the reviewers for their assistance in evaluating this paper.

\section{References}

Béghin, C., P. M. E. Décréau, J. Pickett, D. Sundkvist, and B. Lefebvre (2005), Modeling of Cluster's electric antennas in space: Application to plasma diagnostics, Radio Sci., 40, RS6008, doi:10.1029/ 2005RS003264.

Birdsall, C. K., and A. B. Langdon (1985), Plasma Physics via Computer Simulation, McGraw-Hill, New York.

Blomberg, L. G., et al. (2006), MEFISTO: An electric field instrument for BepiColombo/MMO, Adv. Space Res., 38, 672-679, doi:10.1016/j. asr.2005.05.032.

Bonnell, J. W., F. S. Mozer, G. T. Delory, A. J. Hull, R. E. Ergun, C. M. Cully, V. Angelopoulos, and P. R. Harvey (2008), The Electric Field Instrument (EFI) for THEMIS, Space Sci. Rev., 141, 303-341, doi:10.1007/s11214-008-9469-2.

Cartwright, K. L., J. P. Verboncoeur, and C. K. Birdsall (2000), Loading and injection of Maxwellian distributions in particle simulations, J. Comput. Phys., 162, 483-613, doi:10.1006/jcph.2000.6549.

Cully, C. M., R. E. Ergun, and A. I. Eriksson (2007), Electrostatic structure around spacecraft in tenuous plasma, J. Geophys. Res., 112, A09211, doi:10.1029/2007JA012269.

Fahleson, U. (1967), Theory of electric field measurements conducted in the magnetosphere with electric probes, Space Sci. Rev., 7, 238-262.

Gurnett, D. A. (1998), Principles of space plasma wave instrument design, in Measurement Techniques in Space Plasmas - Fields, edited by R. F. Pfaff et al., pp. 121-136, AGU, Washington, D. C.

Gustafsson, G., et al. (1997), The electric field and wave experiment for the Cluster mission, Space Sci. Rev., 79, 137-156, doi:10.1023/ A: 1004975108657

Gustafsson, G., et al. (2001), First results of electric field and density observations by Cluster EFW based on initial months of operation, Ann. Geophys., 19, 1219-1241.

Hershkowitz, N. (1989), How Langmuir probes work, in Plasma Diagnostics, vol. 1, edited by O. Auciello and D. L. Flamm, pp. 113-184, Academic, San Diego, Calif.

Hockney, R. W., and J. W. Eastwood (1981), Computer Simulation Using Particles, McGraw-Hill, New York.

Miyake, Y., and H. Usui (2009), New electromagnetic particle simulation code for the analysis of spacecraft-plasma interactions, Phys. Plasmas, 16, 062904, doi:10.1063/1.3147922.

Miyake, Y., H. Usui, H. Kojima, Y. Omura, and H. Matsumoto (2008), Electromagnetic particle-in-cell simulation on the impedance of a dipole antenna surrounded by an ion sheath, Radio Sci., 43, RS3004, doi:10.1029/2007RS003707.

Nakashima, H., Y. Miyake, Y. Omura, and H. Usui (2009), OhHelp: A scalable domain-decomposing dynamic load balancing for particle-incell simulations, Proceedings of the 23rd International Conference on Supercomputing, pp. 90-99, Assoc. Comput. Mach., New York, doi:10.1145/1542275.1542293.

Pedersen, A., C. A. Cattell, C.-G. Fälthammar, V. Formisano, P.-A. Lindqvist, F. Mozer, and R. Torbert (1984), Quasistatic electric field measurements with spherical double probes on the GEOS and ISEE satellites, Space Sci. Rev., 37, 269-312, doi:10.1007/BF00226365.

Pedersen, A., F. Mozer, and G. Gustafsson (1998), Electric field measurements in a tenuous plasma with spherical double probes, in Measurement Techniques in Space Plasmas - Fields, edited by R. F. Pfaff et al., pp. 1-12, AGU, Washington, D. C. 
Scudder, J. D., X. Cao, and F. S. Mozer (2000), Photoemission currentspacecraft voltage relation: Key to routine, quantitative low-energy plasma measurements, J. Geophys. Res., 105, 21,281-21,294, doi:10.1029/1999JA900423.

Smith, J. R., N. Hershkowitz, and P. Coakley (1979), Inflection-point method of interpreting emissive probe characteristics, Rev. Sci. Instrum., 50, 210-218.

Taflove, A. (1995), Computational Electrodynamics: The Finite-DifferenceTime-Domain Method, Artech House, Norwood, Mass.
Tsuruda, K., H. Hayakawa, M. Nakamura, T. Okada, A. Matsuoka, F. S. Mozer, and R. Schmidt (1994), Electric field measurements on the GEOTAIL satellite, J. Geomagn. Geoelectr., 46, 693-711.

H. Kojima, Research Institute for Sustainable Humanosphere, Kyoto University, Gokasho, Uji, Kyoto 611-0011, Japan.

Y. Miyake, Academic Center for Computing and Media Studies, Kyoto University, Yoshida-Honmachi, Sakyo-ku, Kyoto 606-8501, Japan. (y-miyake@media.kyoto-u.ac.jp)

H. Usui, Graduate School of System Informatics, Kobe University, Rokkodai-cho, Nada-ku, Kobe 657-8501, Japan. 